\title{
Gestión, Procesos Y Calidad Universitaria: Caso Ecuatoriano
}

\author{
Carlos Ricaurte Yépez \\ José Manuel Pozo PhD
}

Docente - Investigador, Escuela Superior Politécnica de Chimborazo

Facultad de Recursos Naturales, Riobamba - Ecuador

Profesor Titular, Universidad de La Habana, Habana - Cuba

Doi:10.19044/esj.2018.v14n34p195 URL:http://dx.doi.org/10.19044/esj.2018.v14n34p195

\begin{abstract}
The objective of this study was to conduct a critical analysis of university management; for this, secondary information was collected from various bibliographic sources, and the respective analysis and contributions were carried out based on the knowledge acquired. The article deals with the currents and administrative theories, principles about the quality, functions and processes that are carried out in the university and the evolution of this as organizational and academic structure, within a determined historical context, management models, in relation to the management process.
\end{abstract}

Keywords: University, management, processes, quality.

\section{Resumen}

El objetivo de este estudio fue realizar un análisis crítico sobre la gestión universitaria; para ello se levantó información secundaria de diversas fuentes bibliográficas, y se procedió a realizar el respectivo análisis y aportes con base a los conocimientos adquiridos. El artículo trata sobre las corrientes y teorías administrativas, principios sobre la calidad, funciones y procesos que se realizan en la universidad y la evolución de esta como estructura organizativa y académica, dentro de un contexto histórico determinado, modelos de gestión, en relación con los procesos de gestión.

Palabras claves: Universidad, gestión, procesos, calidad.

\section{Introducción}

Los profundos cambios económicos, políticos y sociales desarrollados en el Ecuador en la última década, han generado la necesidad de transformar la educación en todos sus niveles, y en especial la universitaria, por el papel 
que cumple en los procesos de cambio y modernización de la sociedad. Para poder responder a este desafío, se requiere una administración de la educación de calidad, innovadora, construida y ejercida colectivamente, capaz de promover una universidad de puertas abiertas, que responda a los problemas de la sociedad, una universidad que genere ciencia y tecnología. Una universidad que define y articula adecuadamente sus funciones, que entienda que los procesos universitarios fundamentales son la docencia, la investigación y la vinculación con la sociedad y que los procesos administrativos son de apoyo.

Para comprender el problema se debe partir de una revisión de las raíces históricas y tradiciones culturales de la educación latinoamericana y ecuatoriana, y a partir de ahí analizar las propuestas de gestión administrativa en el ámbito de la educación universitaria, para ello se debe examinar la orientación epistemológica y los límites de los fundamentos filosóficos y sociológicos de los paradigmas dominantes en la administración de la educación contemporánea (Ayala 2014). Es importante identificar y analizar algunos de los actuales desafíos conceptuales y de la gestión de la universidad, con la finalidad de desarrollar una perspectiva democrática de administración universitaria, capaz de lograr una educación de calidad para todos, con énfasis en la aplicación de los conceptos de libertad, equidad y participación en la universidad y en la sociedad como un todo. Una educación liberadora, una universidad transformadora, al servicio de la sociedad (Sander 2007).

El proceso de evolución histórica de la universidad en Latinoamérica y en el Ecuador, las corrientes del pensamiento, lo que ha significado la universidad como organización y su relación con el modo de producción imperante, son elementos que deben ser analizados para comprender el problema en su real dimensión. Un hecho es que América Latina no es equitativa el continente más inequitativo del mundo, la universidad lejos de jugar un papel orientador de la conducción del estado, se convirtió por siglos en el proveedor de los profesionales que requería el sistema para su consolidación (Sander 2007).

Es importante comprender lo que implica la administración, la organización de la universidad, cuáles son sus funciones, cómo enfrenta la problemática social, es decir, entender que la educación tiene una connotación política, que debe ser abordada desde la administración de la universidad. En este sentido, la calidad constituye un elemento sustantivo en el quehacer universitario, por lo que se debe analizar esta variable desde una perspectiva diferente a la concebida en la empresa capitalista, y más bien enfocarla desde la perspectiva de la prestación de un servicio público, que además es un derecho de los seres humanos consagrado por las Naciones Unidas (León, 2008). 
Al tratar sobre los procesos que se realizan en la universidad se debe entender cuáles son los procesos gobernantes y misionales, es decir aquellos que tienen que ver con el cumplimiento de la misión de las universidades, esto es la formación profesional, la investigación científica y la vinculación con la colectividad, los que en su conjunto se definen como procesos académicos. Los procesos, relacionados con la gestión administrativa, se conocen como procesos habilitantes o de apoyo, y permiten o soportan a los procesos misionales y gobernantes, por tanto, las universidades dentro de su funcionamiento deben procurar que los procesos relacionados con la gestión administrativa (recursos humanos, materiales, financieros, tecnológicos) deban ser eficientes en su cumplimiento y a la vez, que deben ser suficientes, única forma de cumplir con los objetivos institucionales.

En el Ecuador, el Gobierno del Presidente Correa (2007 - 2017), se realizó una profunda reforma del sistema de educación superior, buscando un modelo alternativo al sistema capitalista de producción, procurando lograr que la universidad se modernizará, dotándole de los recursos económicos suficientes para que pueda cumplir sus objetivos, con miras a la construcción de lo que se ha denominado la sociedad del conocimiento, por lo que se hace necesario una revisión de las formas organizativas y de gestión de las universidades, que les permitan ponerse a tono con los cambios generados y dar respuesta a las demandas de la sociedad.

La universidad ecuatoriana, enfrenta recientes procesos de evaluación y acreditación, como parte de la política del estado por elevar los niveles de calidad en las instituciones de educación superior. La Escuela Superior Politécnica de Chimborazo (ESPOCH) debe enfrentar el reto de un nuevo proceso de acreditación institucional y la acreditación de sus carreras, conforme a las regulaciones establecidas por el Estado. La gestión debe responder a indicadores de desempeño que garanticen la calidad de la educación superior, además la universidad debe dar una efectiva respuesta a las necesidades sociales, desarrollo de sus procesos con pertinencia, por cuanto la disponibilidad de recursos económicos por sí solo, no garantiza una educación de calidad, por lo que se vuelve importante una articulación de los procesos académicos y administrativos con las funciones de la universidad.

\section{Método}

El levantamiento de información secundaria se realizó mediante la revisión documental de bibliografía de diversas fuentes literarias y metodológicas especializadas sobre estudios elaborados acerca la gestión universitaria. Se recurre a fuentes primarias mediante entrevistas a informantes calificados para recoger información sobre la situación del sistema de educación superior en el Ecuador. 


\section{Resultados}

La gestión universitaria, contexto histórico

Es necesario contextualizar el estudio de la gestión académica universitaria en América Latina, examinándola en el ámbito de su historia política, económica, social y cultural, con lo que se busca entender al actual estado del conocimiento en el campo de la administración de la educación como el resultado de un largo proceso de construcción histórica, marcada por una serie de acontecimientos generados es nuestras sociedades e impuestos desde las metrópolis y los organismos de desarrollo, cooperación y ayuda internacional, como parte de una historia inconclusa que se continua escribiendo.

Para Bruner (2004), el proceso de gestación de la actual estructura de la gestión universitaria en Latinoamérica, se divide en cinco enfoques conceptuales y analíticos diferentes mismos que se describen a continuación:

- El enfoque jurídico que dominó la gestión de la educación durante el período colonial, con su carácter normativo y su pensamiento deductivo;

- El enfoque tecnocrático del movimiento científico, gerencial y burocrático de la escuela clásica de administración de inicios del siglo $\mathrm{XX}$, con la lógica económica que caracterizó el proceso de consolidación de la Revolución Industrial;

- El enfoque conductista de la escuela psicosociológica de los años treinta y cuarenta que implementó la utilización de la teoría del sistema social en la organización y gestión de la educación;

- El enfoque desarrollista, de naturaleza modernizadora, concebido en el ámbito de la teoría política comparada que floreció en la posguerra;

- El enfoque sociológico de los autores latinoamericanos de las últimas décadas, preocupados con la concepción de teorías sociológicas y soluciones educativas para satisfacer las necesidades y aspiraciones de la sociedad latinoamericana.

Según Sander B, (2007), la trayectoria histórica del pensamiento administrativo en la educación en América Latina se basa en el movimiento teórico dominante de las ciencias sociales aplicadas. El estudio de las nuevas tendencias en la gestión educativa, se inscribe en ese movimiento y debe beneficiarse de las lecciones del pasado, comprometerse con la solución de los problemas del presente $\mathrm{y}$ anticiparse a las necesidades y aspiraciones del futuro.

\section{Orígenes de la universidad latinoamericana}

La creación de universidades en América Latina bajo la concepción occidental se inicia en la colonia, estableciéndose en 1538 la primera universidad en Santo Domingo, hoy República Dominicana. En 1551 se crean las universidades de Lima y México; más adelante en 1636, cuando se funda 
Harvard, existían 13 universidades en la región, posteriormente llegaron a 31 cuando se dio la independencia (Banco Central del Ecuador, Pensamiento Universitario Ecuatoriano, 1988). La organización de las universidades reflejó el modelo medieval europeo, asociándose con los poderes de la realeza y de la iglesia, posteriormente con la independencia se introduce una nueva idea de universidad, acorde con el surgimiento de las nuevas repúblicas y con la misión de promover la educación, formar profesionales y desarrollar disciplinas académicas.

Para Armengol (2014) el modelo de universidad latinoamericana imita el concepto napoleónico del siglo XIX, según el cual la enseñanza profesional se separa de los centros de generación del conocimiento, exclusivamente académicos y científicos. Frente a este modelo, otra concepción se originó en Alemania, con la emergencia de la universidad de investigación, donde la enseñanza se combinó con la generación del conocimiento y la ciencia. Esta revolución académica se consolidó en Europa en el siglo XIX y luego en EE.UU, con el auge de las tecnologías y el desarrollo de nuevas dinámicas económicas, cuya base era, y sigue siendo la asociación del conocimiento con su aplicación práctica. Posteriormente, en el siglo XX, la investigación científica y tecnológica en los países desarrollados terminó por ligarse directamente con el crecimiento económico, considerando por medio del modelo universitario que la generación, transmisión y aplicación de conocimientos eran actividades inseparables, respondiendo a una dinámica económica que fundamenta y convierte a la universidad en el proveedor de la mano de obra especializada que necesita la industria.

De acuerdo con Sander (2007), el proceso endógeno de producción de conocimientos en las universidades y centros de investigación de los países en desarrollo, responde y es alimentado por las demandas del crecimiento económico y social, es por ello que en el siglo XXI, los modelos universitarios de los llamados países en vías de desarrollo se encaminan en esta dirección. La tendencia es pasar de la universidad de investigación a la universidad empresarial, orientada a responder al mercado, a las necesidades del sector productivo, obtener financiamiento de las empresas, y contribuir con el crecimiento económico.

\section{Problemática de la Universidad en América Latina}

La historia del proceso de modernización de las universidades en América Latina muestra las evidentes contradicciones de políticas que fracasaron en el intento de implantar y llevar a la práctica principios y modelos que se revelaron imposibles de aplicar, porque no se correspondían con el nivel de nuestras fuerzas y capacidades sociales y productivas. La universidad desde la independencia buscó democratizar la educación, bajo los principios de humanismo e igualdad europeos. La intención de profesionalizar a la 
población en países como Brasil, Argentina, Colombia, Perú, México o Ecuador, que no contaban, con un mínimo de masa burguesa capaz de acceder a la educación superior, y donde no existía un proceso de industrialización, ni una demanda real que justificara este esfuerzo, resultó en la formación de universidades que sólo atendían a una élite, una minoría privilegiada, siempre de cara a Europa y los centros de desarrollo.

Las universidades latinoamericanas del siglo XIX, no se enfocaron en las funciones de investigación y generación de conocimientos, privilegiaron la enseñanza y formación de profesionales, siguiendo el modelo francés napoleónico que no respondía a las necesidades de las repúblicas nacientes.

La imposición de modelos de desarrollo excluyentes, producto de la consolidación del sistema capitalista de producción, genera una agudización de las contradicciones en Latinoamérica, evidenciando que la universidad republicana no había logrado romper con los esquemas coloniales de carácter feudal. El resultado a inicios del siglo XX fue otro intento de modernización, hacia una verdadera democratización de las estructuras universitarias, surgiendo con ello el movimiento de reforma universitaria, que comenzó en Argentina con protestas estudiantiles que denunciaron la permanencia de estructuras clasistas y oligárquicas que no responden a los procesos de modernización social, concretándose con la reforma de Córdoba de 1918, que se extendió rápidamente en la región.

Las reformas del siglo XX no apuntaron a definir el verdadero rol de las universidades en la producción del conocimiento y su función social, no se centran en la creciente importancia de la ciencia para el desarrollo técnicoproductivo, ni en cambiar los modelos económicos dependientes y progresar hacia la industrialización. Los esfuerzos por convertir a las universidades en motores eficientes de la democratización social y cultural, y del desarrollo no fueron suficientes, las desigualdades sociales crecieron en nuestros países, teniendo un acceso al conocimiento inequitativo.

\section{La búsqueda de nuevos modelos de gestión, la universidad del siglo XXI}

En la gestión de la educación, las experiencias existentes destacan la importancia de la democracia como forma de gobierno y la participación como estrategia administrativa. En la universidad, existe un consenso sobre la importancia de la calidad de la gestión y de sus procesos. Sin embargo, no existe consenso sobre la naturaleza de la calidad y de sus estrategias de aplicación en distintas especialidades del quehacer humano.

Los resultados de los estudios desarrollados en los últimos años a nivel latinoamericano, apuntan a la formulación de un nuevo paradigma multidimensional de administración de la educación, fundamentado en la desconstrucción y la reconstrucción de los conocimientos pedagógicos y administrativos acumulados en el curso de la historia de la educación 
latinoamericana (Noboa, 2012). Esa reconstrucción teórica exige un amplio enfoque interdisciplinario para tratar de explicar los dominios de las influencias económicas, políticas, culturales y pedagógicas en la organización y administración de la educación en el hemisferio occidental.

El paradigma multidimensional, es un modelo en vías de construcción; resultante de un proceso de aprendizaje permanente y de un esfuerzo de superación intelectual, buscando satisfacer necesidades de las universidades. Los esfuerzos de construcción teórica se apoyan en el convencimiento de que la evaluación de las bases epistemológicas que han inspirado las teorías organizativas y administrativas adoptadas históricamente en la educación permiten examinar el papel de la mediación administrativa en la organización y la gestión de los sistemas de enseñanza Ayala (2014), indica que un proceso de evaluación epistemológica no admite concesiones académicas ni establece condiciones teóricas, la única condición previa se relaciona con los valores éticos establecidos en conjunto por la ciudadanía, como la libertad y la equidad, que deben orientar la formación de una sociedad capaz de promover la calidad de vida humana individual y colectiva.

Esta evaluación revela como la tradicional administración tecnoburocrática de orientación positivista y funcionalista adoptada en el sector público y en la educación latinoamericana tiene como objetivo alcanzar el orden y el progreso racional, la reproducción estructural y cultural, la cohesión social y la integración funcional en la universidad y en la sociedad (Gramsci, A. 1987). Además, se muestra que la administración tecno-burocrática tiene componentes autoritarios que limitan el espacio para la promoción de la libertad individual y la equidad social; su carácter normativo y jerárquico inhibe la creatividad y dificulta la participación ciudadana.

En una versión superadora de las teorías tradicionales de gestión educativa adoptadas en la sociedad occidental, se desarrolló una construcción integradora de administración de la educación, apoyada en los principios del liberalismo social adoptado actualmente por las fuerzas liberales abiertas a la problemática social. Las décadas del 70 y 80 fueron testigos de una nueva efervescencia intelectual liderada por los teóricos críticos en las universidades y asociaciones de educadores de América Latina, basado en la literatura europea y norteamericana, que dio origen a una teoría crítica de educación y de gestión educativa, cuya influencia político-pedagógica no debe ser subestimada. En un esfuerzo de superación intelectual, los pensadores críticos tratan de concebir, partiendo del legado histórico sobre la construcción dialógica de administración de la educación que rescata el criterio de totalidad y se identifica con los principios adoptados por las fuerzas comprometidas con la reconstrucción de la perspectiva socialista y de la naturaleza de civilización humana que ella implica. 
La evaluación de la experiencia educativa latinoamericana revela que todos necesitamos aprender, con la meta colectiva de concebir perspectivas intelectuales socialmente válidas y culturalmente relevantes que sirvan de guía para la investigación y la práctica en la administración de la educación, además incorporar y potenciar la formación de profesionales emprendedores, para el desarrollo de un sistema económico que incorpore tecnología y conocimientos a los productos primarios, que genere valor añadido a la producción, que contribuya al cambio de la matriz productiva y energética, son varios de los retos de la universidad en el siglo XXI, la que además deberá afrontar en condiciones adecuadas los procesos de evaluación que se implementan desde el estado y dar una efectiva respuesta a las demandas de la sociedad.

\section{La universidad en el Ecuador}

Los orígenes de la universidad ecuatoriana, según se expresa en el libro Pensamiento Universitario Ecuatoriano (1988), data de 1622, cuando por autorización del Rey Felipe IV, los jesuitas fundan la Real y Pontificia Universidad de San Gregorio, posteriormente en 1681 los dominicos crean la Universidad de Santo Tomas de Aquino; en 1822 el claustro universitario decide cambiar la estructura y funcionamiento de la antigua universidad clerical, a una universidad que responda a la nueva estructura del estado.

En 1826, la Gran Colombia en el Congreso de Cundinamarca, decide que habrá universidades centrales en las capitales de los departamentos de Cundinamarca, Venezuela y Ecuador, nace así la universidad estatal y se crean profesiones liberales como derecho, medicina, literatura, y las tradicionales filosofías, ciencia eclesiástica. Desde el nacimiento de la República en 1830, hasta mediados del siglo XX, en el Ecuador existieron 5 universidades públicas (Universidad Central, Universidad de Guayaquil, Universidad de Cuenca, Universidad de Loja y Escuela Politécnica Nacional), configurando un país agrario y rural, en el que el centro de poder político y económico estaba en Quito y Guayaquil, hecho que motivó una gran migración de jóvenes a las grandes urbes, buscando acceder a la universidad como única forma de ascenso social y búsqueda de oportunidades. Entre 1945 y 1975 se crean en el Ecuador 20 universidades, como resultado de la presión que se ejercía desde ciudades intermedias por contar con un centro de estudios superiores (Riobamba, Ambato, Ibarra, Babahoyo Machala, Portoviejo, entre otras), además, surgen cinco universidades particulares, todas de congregaciones religiosas.

La Comisión Económica para América Latina, CEPAL (1972), indica que en las décadas del 60 y 70, se producen transformaciones en la estructura social y económica del país, el modelo agrario - exportador empieza a ser reemplazado por el de sustitución de importaciones, crece en gran dimensión la economía por efectos del boom petrolero, cambia la estructura poblacional, 
con la masiva migración campo-ciudad; es el inicio de la incorporación de la mujer al mercado laboral asumiendo nuevos roles, factores que producen una enorme expansión en la matrícula universitaria. Es el periodo de transformación del aparato productivo, de un importante crecimiento económico y una acelerada modernización del país, generando la necesidad del aparato productivo y burocrático de profesionales en áreas técnicas, administrativas y gerenciales, que deben ser cubiertas por las universidades.

Entre 1980 y 2005 se crean cerca de 40 universidades, es el periodo de la universidad - empresa, la consolidación del neoliberalismo, que genera oportunidades de negocio, y entre sus preceptos está el privatizar todo y considerar a la educación como una mercancía; surgiendo grupos de empresarios deseosos de invertir y generar utilidades mediante la oferta de estudios superiores, prima la obtención de beneficios sobre cualquier precepto de calidad, proliferan universidades y carreras, se diversifican los títulos, se instaura el facilismo, lo que lleva a una descomposición total, del rol y papel de la universidad. A partir del año 2008, se inicia un proceso de cambios en la educación superior, se establecen sistemas de evaluación y acreditación de las universidades, incrementando la asignación de recursos económicos, estandarizando la nomenclatura de grados y títulos y se implementan los exámenes de admisión en el sistema de educación superior.

\section{Universidad, gestión y calidad}

Es importante plantear algunos desafíos conceptuales y analíticos en el campo de la administración pública y la gestión educativa en el contexto del nuevo orden económico y político internacional.

Bruner (2008), manifiesta que están en debate algunos temas, como gestión educativa y desarrollo en un contexto de interdependencia internacional; formación humana sostenible y gerencia social; teoría crítica y participación ciudadana en la gestión de la educación; relevancia de la escuela y efectividad de la comunidad en el contexto de las promesas y falacias de la descentralización administrativa, así como la gestión democrática para una educación de calidad para todos. Para administrar esa crisis estructural, muchos dirigentes, especialmente en el sector público, buscan soluciones en los postulados de Taylor (1946) y Emerson (1948), padres de la eficiencia económica en la teoría administrativa, observando con ello durante la década de los ochenta una creciente utilización de prácticas neo-tayloristas en la administración del Estado y en la gestión de las instituciones sociales en general. Se acentúa la producción académica para definir la naturaleza de la calidad en la administración de los servicios públicos y en la gestión de la educación en particular. Se observa que, a pesar de la fascinación que ejerce el concepto de calidad total, existe una falta de definición respecto a su 
utilización en las diversas organizaciones sociales en función de la variedad de fines, de servicios y de intereses creados.

Por la propia esencia de la educación, el concepto de calidad total, de la moderna organización industrial no puede ser transferido automáticamente a la organización y la gestión de la educación. No se debe aplicar conceptos e instrumentos de gestión y evaluación uniformes, de carácter universal, para realizar el monitoreo de instituciones y actividades heterogéneas desde lo ideológico, organizativo y funcional. Estas observaciones no buscan reducir la importancia de la eficiencia y la racionalidad como indicadores de calidad en la administración de los servicios educativos; por el contrario, debido a la importancia central de la calidad en la gestión de la educación, la primera preocupación se relaciona con la necesidad de definir correctamente la naturaleza de la calidad de educación y de su proceso administrativo.

Cuando se trata de la prestación de servicios públicos, definidos como estratégicos para el desarrollo de la sociedad, se debe entender que no se trata de clientes o consumidores, sino de ciudadanos con deberes y derechos. Muchas veces la elección de los servicios en el sector público es difícil, cuando no imposible, por falta de opciones alternativas, lo que sugiere la necesidad de desarrollar otras alternativas de participación en la gestión para la mejora de la calidad de la educación.

El primer requisito para poder abordar correctamente el tema de la calidad de la administración de la educación es rescatar la especificidad de la educación y la naturaleza peculiar de la calidad de la educación. La educación, definida como instancia de construcción y distribución del conocimiento socialmente válido y culturalmente relevante para la ciudadanía. La calidad de la educación puede definirse a partir de diversas perspectivas conceptuales y dimensiones analíticas. Es posible valorar la educación en términos políticos y académicos. La calidad política refleja su capacidad por alcanzar los fines y objetivos políticos y culturales de la sociedad. La calidad académica define el nivel de eficiencia y eficacia de los métodos y tecnologías utilizados en el proceso educativo (Druker 1973). También es posible valorar la educación en términos individuales y colectivos. La calidad individual define la contribución de la educación al desarrollo de la libertad subjetiva y del interés personal. La calidad colectiva mide la contribución de la educación a la promoción de la equidad social y del bien común (Brunner 2008).

La bibliografía pedagógica latinoamericana destaca el carácter central de la preocupación por la calidad de la educación. En esa línea, la contribución de Tadesco (1995), está centrada en la construcción de opciones educativas con elevados niveles de calidad para todos, procurando combinar la excelencia académica con la democratización del acceso a los conocimientos socialmente significativos. Aguerrondo (1993), concentra su discusión en la intersección de los aspectos político-ideológicos y de las opciones técnico-pedagógicas en 
la evaluación de la calidad de los servicios educativos y de la gestión para la mejora de la calidad de la educación. Frigerio y Poggi, (2000) adoptan un concepto comprensivo de calidad de la educación, definiéndola como la integración de la calidad de la organización institucional, de los recursos humanos y financieros, de la gestión de la enseñanza, de la propuesta curricular y didáctica, del proceso educativo y sus resultados en términos de aprendizaje. Schmelkes (2006), hace una contribución llamativa sobre calidad de la educación en la escuela básica, que permite reflexionar sobre los límites e implicaciones de los nuevos enfoques de administración en la educación contemporánea.

En diversos estudios latinoamericanos sobre la calidad de la educación y su relación con la calidad de la gestión educativa existe una preocupación prioritaria con la equidad y la relevancia social de la educación y del conocimiento para la ciudadanía. Braslavsky y Tiramonti (1990), resumen esa preocupación cuando afirman que "la búsqueda de la calidad no debe hacerse a expensas de la equidad". Hallack (1989), defiende una propuesta de administración escolar capaz de articular creativamente los ideales de calidad y equidad en la prestación efectiva de los servicios educativos. Namo de Mello (2010) revela la misma preocupación con la calidad y la equidad en su propuesta de una escuela pública de calidad para todos y en su discusión de las nuevas exigencias de una gestión educativa construida a nivel local, que permita incorporar necesidades desiguales y trabajar sobre las mismas a lo largo del proceso de escolarización, a fin de asegurar el acceso al conocimiento y la satisfacción de las necesidades básicas de aprendizaje para todos.

Calidad de educación para todos es, en realidad, el consenso políticopedagógico de alcance internacional más importante adoptado por los gobiernos en las puertas del nuevo milenio. El compromiso con la universalización de una educación básica de calidad fue reasumido en la Conferencia Mundial de educación para todos, realizada en Jomtien, Tailandia (1990), bajo el auspicio de UNESCO, UNICEF, PNUD y el Banco Mundial. Desde entonces, se observan nuevos esfuerzos nacionales en la educación latinoamericana, cuyos gobiernos al firmar la declaración, asumieron el compromiso de desarrollar un plan decenal para la universalización de una educación básica de calidad. En el ámbito político del sistema interamericano, el ideal de educación de calidad en todos los niveles de enseñanza, y la democratización de la gestión educativa integran el plan de acción adoptado por los Jefes de Estado y de Gobierno en la Cumbre de las Américas (Miami, 1994).

El concepto de calidad de la educación implica desarrollar un ambiente cualitativo de trabajo en las organizaciones educativas, mediante la institucionalización de conceptos y prácticas, tanto técnicas como administrativas, capaces de promover la formación humana sostenible y la 
calidad de vida de estudiantes, profesores y funcionarios. En términos operativos, esos conceptos sugieren organizar las instituciones educativas y sus procesos administrativos y pedagógicos con racionalidad y pertinencia, para que puedan contribuir efectivamente a la construcción y distribución del conocimiento y a la prestación de servicios relevantes para la comunidad y la sociedad como un todo (León, 2012).

Es importante relacionar estos conceptos con lo propuesto por Demming (1981) quien plantea el ciclo de mejora continua: planificar, hacer, verificar y actuar, como un referente para la implementación de este tipo de procesos en la universidad, elemento que es recogido como básico en los procesos de certificación International Organization for Standardization (ISO). La calidad de la actividad educativa se encuentra necesariamente relacionada con la calidad de los trabajadores de la educación; están estrechamente vinculadas a la calidad de los elementos organizativos y de los procesos técnicos y administrativos en el lugar de trabajo, lo que incluye el aula, la universidad y las instancias superiores.

La Junta de Acreditación Nacional (2009), organismo oficial que rige el proceso de acreditación de carreras y programas universitarios en Cuba, indica que el patrón de calidad persigue identificar un modelo ideal al cual deben aproximarse las carreras que se desarrollan en las Consejo de Educación Superior (CES), el que define el deber ser del proceso de formación de profesionales y define a la calidad como la conjunción de la excelencia académica y revolucionaria, con pertinencia social. Por lo tanto, los procedimientos administrativos, los procesos técnicos y la misión de las instituciones educativas deben ser concebidos como componentes articulados de un paradigma comprensivo de gestión para mejorar la calidad de la educación para todos.

De la calidad de la gestión así concebida y ejercida depende, en gran parte, la capacidad institucional para construir y distribuir el conocimiento, definido como el factor clave de los nuevos niveles de desarrollo y de la nueva estructura de relaciones sociales, tanto a nivel nacional como en el ámbito internacional.

\section{¿Hacia dónde debe ir la universidad latinoamericana?}

A partir de esta revisión histórica, se infiere que se ha fallado en la comprensión de la problemática educativa, por lo que cabe preguntarse: ¿Qué es y para qué sirve una universidad en Latinoamérica?, ¿A quién se dirigen sus servicios?, ¿Cuáles son sus funciones y cómo se evalúa su funcionamiento?, ¿Qué tipo de profesionales debe formar la universidad, y de qué manera estos contribuirán al desarrollo productivo y social de sus sociedades? 
El concepto que parece más adecuado para definir lo que debería ser actualmente una universidad latinoamericana, es el que conjuga en una institución: niveles de excelencia y eficiencia, evaluados en resultados concretos, en tres direcciones fundamentales: en la formación de los profesionales para atender las necesidades presentes y previsibles para el desarrollo del país; en las actividades de investigación y generación de conocimientos, desarrollo de conocimiento relativo a valores culturales, científicos y tecnológicos universales, y como actividades más adaptadas a nuestra realidad latinoamericana actual, en el aprendizaje, adaptación y aplicación nacional de tecnologías existentes; y en respuestas concretas a las demandas del entorno, tanto a nivel nacional como regional, con estrategias de inserción en la economía global. Son los tres ejes de la moderna acción universitaria: formación, investigación y extensión.

En el escenario actual, los ejes de acción universitaria son los mismos y apuntan a las mismas direcciones, tanto en países desarrollados como en los países latinoamericanos, los objetivos de crecimiento son diferentes, así como lo son sus modelos sociales, sus capacidades y el desarrollo de fuerzas y relaciones productivas. Por consiguiente, también serán distintos los planes, estrategias, acciones, formas organizativas, y evaluación de procesos, que se deban implantar para alcanzarlos. En lugar de enfocarse en esos objetivos de fondo y las estrategias para cumplirlos, las políticas universitarias en Latinoamérica parecen haberse limitado a considerar que, para alcanzarlos bastaba con implantar enunciados, formas y estructuras de funcionamiento, copiando modelos, planes, programas, esquemas organizativos, y hasta estándares de evaluación de países desarrollados, enfrentando, como consecuencia, las contradicciones inevitables que se presentan al tratar de alcanzar objetivos ideales, anunciados en cada sucesiva gestión de gobierno universitario.

Por un lado, instituciones autónomas en el plano político, pero, que, al mismo tiempo, mantienen una casi completa dependencia económica de los recursos del Estado, en consecuencia, están inevitablemente sujetas a la inestabilidad, actuación y cambio de los gobiernos de turno; por otro lado, realizar investigación científica de punta y producir nuevos conocimientos que sean valorados internacionalmente, con escasos recursos e insatisfactorios resultados, que además, están generalmente desvinculados de la demanda nacional, lo que hace que esta producción de conocimientos sea considerada irrelevante y poco valorada socialmente.

Y por último, acceso igualitario y democrático a la formación que imparten, objetivo poco factible de cumplir en la región con las mayores desigualdades sociales del planeta, y con actividades orientadas a obtener un relativo impacto en el desarrollo económico y social, dada la escasa vinculación de las universidades con los sectores productivos y sociales 
Por lo que se hace necesario desarrollar una serie de acciones a nivel de las universidades y en coordinación con el estado, que les permita mejorar sus sistemas administrativos y de gestión.

Dentro del enfoque de sistemas se concibe a la organización como una estructura única, unida, dirigida, de partes interrelacionadas. Este enfoque para administrar, en lugar de abordar los diversos segmentos de una organización por separado, piensa que la organización es un sistema único, que tiene un propósito y está compuesto por partes que se interrelacionan. Esta posición permite a los directivos mirar a la organización como un todo y como parte del ambiente externo. Se indica que la actividad de un segmento de la organización afecta en diferentes grados, a todos los segmentos. Para entender este enfoque, se ha tomado en cuenta la Teoría de Sistemas, propuesta en 1956 por el biólogo Ludwing Von Bertanlanffy, quien manifiesta que el análisis del conjunto, de sus componentes y relaciones, debe hacerse a través de la relación entre los mismos.

Este enfoque es extendido a otras áreas del saber, entre ellas las Ciencias Económicas, al respecto, Rodríguez (1992) sintetiza las características más importantes de la organización como sistema: la organización puede considerarse como un sistema abierto; la organización se considera como un conjunto de elementos interrelacionados e interactuantes interna y externamente; la organización está articulada de forma jerárquica, cada sistema es un supra-sistema para otro subsistema a la que contiene como elemento; la organización es más que la suma de sus partes.

Lo que implica pensar en la forma como se deben definir y articular las funciones con los procesos dentro de la universidad, y la forma como la organización define la metodología para su implementación.

\section{Los procesos en la educación superior. Metodología para su análisis}

Evans \& Lindsay (2000) conceptualizan al proceso como una secuencia de actividades que tienen la finalidad de lograr algún resultado, generalmente crear un valor agregado para el cliente. Pozo \& Rodríguez (2015) indican que, el proceso es un conjunto de actividades interrelacionadas, que persiguen la creación de valor y que su salida final es la conformación de un bien o servicio para un cliente de la organización.

Al ser una de las funciones de la universidad preservar, desarrollar y difundir la cultura universal y nacional, su influencia se hace más trascendente en la medida en que las sociedades tengan un mayor desarrollo, pero la pertinencia e impacto de la universidad como preservadora, desarrolladora y difusora de la cultura no se logra de manera espontánea ni depende sólo de criterios subjetivos, es consecuencia de la aplicación de la ciencia, al estudio desde dentro y hacia dentro del proceso (Fuentes, 2003). La relación sociedad - universidad se expresa mediante múltiples manifestaciones que se concretan 
en los modos de actuación universitarios para satisfacer las necesidades sociales (León, 2012). El vínculo entre la universidad y el contexto social es permanente, en tanto es consustancial a los procesos que en el seno de la universidad se desarrollan.

Los procesos universitarios, por su propia naturaleza, tienen al ser humano en su centro, por ello son identificados como procesos conscientes, de naturaleza holística y dialéctica. Por otra parte, los procesos se dan interrelacionados y se establecen regularidades que determinan las cualidades de todo como sistema, y adquieren significación a partir de su mutua vinculación, lo que es expresión de la naturaleza totalizadora que tienen estos procesos.

La especificidad de la preservación de la cultura tiene su máxima connotación en los procesos de formación de los profesionales, su superación y actualización permanente; el desarrollo de la cultura mediante el proceso de investigación científica y de creación en general y la difusión mediante los procesos de vinculación; estos procesos se dan en unidad debido a que la formación de los profesionales conlleva la investigación y a que todo proceso de investigación científica y de creación pasa necesariamente por un proceso de formación, todo lo cual pasa por la difusión de la cultura tanto en el ámbito universitario, como hacia su entorno.

En consecuencia, los objetivos a alcanzar en los procesos universitarios se mueven entre lo profesional y lo investigativo, desde lo productivo a lo creativo. Según León (2012), los procesos universitarios se dan en respuesta a las necesidades sociales que se concretan en la preservación, desarrollo y difusión de la cultura, por lo que en correspondencia con los procesos universitarios, los resultados más significativos de la universidad son los siguientes:

- Profesionales graduados (grado y posgrado), función docencia

- Resultados científicos - técnicos, función investigación

- Aportes complementarios a la comunidad, función vinculación

Guerra \& Meizoso (2012), definen al proceso como, el conjunto de actividades mutuamente relacionadas o que interactúan, las que transforman elementos de entrada (insumos) en resultados (productos); sobre el enfoque por procesos, indican que el resultado se logra con mayor eficiencia si los recursos y las actividades se gestionan como un proceso, y que la organización debe concentrar sus esfuerzos y priorizar la utilización de sus recursos, en los procesos fundamentales.

Para emprender en un enfoque por procesos, es necesaria la utilización de una metodología, por lo que se recurre por su sencillez, lógica y utilidad práctica, a la propuesta por Pozo \& Rodríguez (2015) constante de 7 pasos a saber:

- Identificación de los procesos claves de la organización. 
- Elaboración del Mapa de Proceso de la entidad.

- Confección de las gráficas de proceso.

- Análisis de los procesos.

- Propuestas de mejora.

- Elaboración de fichas de los procesos.

- Propuesta de indicadores para medir el desempeño del proceso mejorado.

A su vez para el análisis y mejora de los procesos se recomienda utilizar la metodología diseñada por la empresa Motorola en 1998, adaptada y aplicada en Cuba por los citados autores, y consta de 10 pasos (e Benito, 2000; Membrado, 2004; Harry y Schroeder, 2004). Esta metodología indica que, para desarrollar un análisis sobre cualquier proceso siguiendo la cadena de valor, es decir, la agregación de valor al producto, en la medida que pasa por las distintas fases o actividades que integran un proceso se dar respuesta a la siguiente lista de interrogantes:

¿Todos los pasos del proceso están organizados en una secuencia lógica?

¿Todos los pasos agregan valor?

¿Puede eliminarse algunos de los pasos a fin de mejorar la calidad?

¿Deben añadirse algunos pasos a fin de mejorar la calidad?

¿Algunos pasos deberían reordenarse?

¿Existe proporcionalidad entre cada uno de los pasos que conforman el proceso?

¿Qué habilidades, equipos y herramientas se requieren en cada uno de los pasos del proceso?

¿Deberán mecanizarse o automatizarse algunos pasos del proceso?

¿En qué punto debe medirse o controlarse la calidad?

¿En qué punto del proceso pueden ocurrir los errores más frecuentes?

Como se puede apreciar las preguntas son claras, sencillas y lógicas, para aplicar la metodología y obtener resultados efectivos se requiere, que una vez que se haya elaborado la gráfica del proceso, se reúna a todos los trabajadores que estén involucrados en su desarrollo y que se proceda a revisar cada una de las preguntas; de aquí se infiere que este es y tiene que ser un trabajo de equipo, donde el obrero o el empleado juegan un papel trascendental. La respuesta a cada interrogante debe obtenerse, sino por consenso sí por mayoría.

Los procesos universitarios de pertinencia e impacto (misionales)

De acuerdo a las funciones esenciales de la universidad establecidas en la Ley Orgánica de Educación Superior (2010), recogidas en el Estatuto de la ESPOCH del año 2013, se identifican y definen 3 tipos de procesos:

- Procesos Gobernantes: Orientan la gestión institucional a través de la formulación de políticas, expedición de directrices, normas, procedimientos, planes estratégicos, acuerdos, resoluciones e instrumentos para el funcionamiento de la organización 
- Procesos agregadores de valor o misionales: Son los que generan el portafolio de productos y servicios, administran y controlan los productos y servicios destinados a usuarios externos, permiten cumplir con la misión institucional, los objetivos estratégicos y constituyen la razón de ser de la institución.

- Procesos habilitantes: Se clasifican en procesos de asesoría y de apoyo, están encaminados a generar productos y servicios de asesoría y apoyo logístico para producir el portafolio de productos institucionales demandados por los procesos gobernantes, agregadores de valor y para sí mismos, viabilizan la gestión.

Los dos primeros procesos son considerados procesos de pertinencia e impacto social de la universidad, pues a través de ellos se da respuesta a las exigencias y demandas de la sociedad. Los procesos de apoyo son considerados de existencia universitaria, pues sin estos no es posible cumplir con la misión de la universidad.

\section{Proceso de docencia, formación de profesionales}

El proceso de formación de profesionales o proceso de grado comprende la formación curricular y extracurricular, que se desarrolla en las instituciones de educación superior. Proceso que se sustenta en un modelo pedagógico de competencias y desarrollo profesional de la ESPOCH del año 2011, como base de la formación de un profesional de amplio perfil, versátil, portador de elevados compromisos profesionales y sociales, con plena conciencia sobre sus deberes y responsabilidades ciudadanas y que, a partir de una profunda formación teórica, de una amplia cultura científica, técnica y humanista, y del desarrollo y sistematización de efectivas habilidades profesionales, fuera capaz de resolver, de manera independiente y creadora, los problemas básicos, más comunes, cotidianos, que se presenten en su perfil ocupacional. Es posible el perfeccionamiento permanente de este modelo cuando adquiere niveles de calidad a través de la excelencia alcanzada por el claustro y la profesionalización de su dirección docente, responsables de la implantación y del desarrollo del modelo y del proceso académico; garantizado por el aseguramiento estatal de la consecuente base material: edificaciones, literatura, laboratorios, talleres y por la proporcionalidad lógica de la relación estudiante - profesor.

Como reflexión cabe plantear si en este proceso se está formando solo profesionales, o si estos tienen una orientación humanista y de compromiso con la sociedad, y si además son capaces de generar nuevas iniciativas de negocios, si pueden ser de base tecnológica, de ser así, el autor concluye manifestando que la universidad está cumpliendo con el encargo social. 


\section{El proceso de formación de postgrado}

Según León (2012), el concepto de calidad en su aplicación a la educación de postgrado resume 2 elementos: pertinencia social y excelencia académica. La pertinencia se refiere a la respuesta que da la universidad a la problemática social, que debe contribuir a resolverse a través de las políticas de postgrado. La idea de excelencia alude a ciertos estándares cualitativos y cuantitativos que la actividad de postgrado debe satisfacer para efectuarse con la calidad requerida. La pertinencia está asociada a las importantes preguntas: ¿postgrado para qué?, ¿posgrado para quién. La excelencia indica que el servicio debe prestarse bajo ciertas condiciones de rigor académico que son cuidadosamente observadas y por ello se refiere al qué y al cómo del postgrado.

Vale resaltar que ambas ideas, pertinencia y excelencia, en su interrelación, son parte del concepto de calidad que el postgrado promueve.

La discusión por la calidad es parte de lo que se llama subsistema de postgrado, cuyas modalidades principales son las especialidades, maestrías y doctorados, niveles que, se caracterizan por la creación de nuevos conocimientos, en consecuencia, es en este nivel donde el problema de la investigación científica se toma como eje, en tanto se pretende desarrollar capacidades para la investigación. Para Norberto Maldonado, ex rector de la ESPOCH en el año 2015, el desarrollo del postgrado y su excelencia es uno de los grandes desafíos de la Educación Superior y requiere de la voluntad institucional que favorezca su atención prioritaria.

\section{El proceso de investigación científica}

La investigación científica y tecnológica tiene un importante papel en la Educación Superior. En primer lugar, adquieren la misión de incidir de forma efectiva en el desarrollo económico y social de las naciones mediante su contribución al proceso de creación científica e innovación tecnológica, como consecuencia de los efectos y repercusión que está teniendo en el mundo, la llamada revolución del conocimiento. Este papel de la ciencia como factor de renovación de la educación superior es reconocido por la UNESCO (1996), cuando señala que cada institución universitaria debe ser una comunidad dedicada a la investigación, la creación y la difusión del conocimiento, al progreso de la ciencia.

Otro aspecto importante del quehacer científico en las universidades, es contribuir al desarrollo y existencia de un sector docente de excelencia como consecuencia de la participación de profesores y estudiantes en el proceso de investigación, siendo una concepción de la educación superior, que no hay verdadera docencia universitaria sin actividad de investigación, ella forma parte del proceso de formación de los estudiantes y tiene un gran valor en la formación profesional. 
Diversos factores resultan significativos para brindar un aseguramiento de la calidad del trabajo de investigación científica. En primer lugar, el establecimiento de una política científica universitaria, en la que se consideran, entre otros aspectos, una precisa política de investigación y organización de la ciencia, así como, la de formación de los recursos humanos, encaminada a garantizar la misión trazada. El establecimiento de programas universitarios de investigación, constituye el eslabón central de la gestión de ciencia y tecnología, al posibilitar dirigir el esfuerzo científico y tecnológico a los sectores y ramas de la economía y de la ciencia, en los que el estado ha establecido prioridades determinantes del desarrollo. Esto se logra mediante la planificación de los proyectos de investigación, sobre la base de obtención de resultados que partan de objetivos de carácter socialmente útiles y de aplicación en las diversas esferas de la economía y de la sociedad.

Otro factor de influencia en la excelencia del producto de investigación científica, constituye el considerable potencial humano de alta calificación y experiencia con que se cuente en las instituciones universitarias. El desarrollo de los servicios de información científico - técnica que garanticen el acceso a las fuentes mundiales de información más novedosas, el equipamiento de los colectivos científicos con técnicas y ordenadores de última generación, son acciones que tienen prioridades. La colaboración científica - técnica internacional con instituciones de alto prestigio, también debe ser parte de la política científica universitaria.

\section{El proceso de vinculación con la sociedad}

La vinculación universidad - sociedad, está considerada como una de las funciones principales del trabajo de la educación superior, y se entiende como el sistema de interacciones universidad - sociedad, que tiene el propósito de promover la cultura en la comunidad universitaria y externa, para contribuir a su desarrollo. La extensión universitaria es una manifestación de la relación dialéctica entre la universidad y la sociedad, se da en el vínculo, cuyo fin es la promoción de la cultura, en el que se establece la relación, entre cultura y elevación del nivel cultural de la sociedad a través de la función extensionista.

La concepción de la extensión universitaria asume a la promoción cultural como su metodología, constituye un elemento esencial para garantizar que pueda ejecutarse y contribuir al cumplimiento de la misión social universitaria en su conjunto; a partir del desarrollo de acciones que se dirigen a la creación de valores culturales, la conservación de los valores, la difusión $\mathrm{y}$ el disfrute.

La vinculación como función y como proceso universitario orienta su proyección al interior de la universidad y hacia la sociedad, que definen las líneas fundamentales de atención en correspondencia con la satisfacción de las necesidades de elevación del desarrollo cultural de la comunidad universitaria 
y de su entorno, resultados que como factor de calidad de la educación determinan su relevancia. Tanto en el orden interno como hacia afuera, la gestión de la extensión se planifica, organiza y ejecuta por medio de proyectos de vinculación.

La extensión universitaria requiere de una estrecha coordinación de los factores externos e internos que participan en el cumplimiento de los objetivos propuestos, y su gestión debe desarrollarse desde la perspectiva del beneficiario por medio de actividades, acciones, tareas, y de la integración de estas en los proyectos de extensión universitaria, los que estarán precedidos del diagnóstico de la realidad y de la identificación del problema o las tendencias de desarrollo. De los resultados de la labor de vinculación depende en buena medida la relevancia o significación de la universidad y como consecuencia el reconocimiento de su grado de desarrollo cualitativo.

\section{Los procesos administrativos}

La gestión integral de la universidad, tiene su soporte en la función administrativa y se entiende como el sistema de interacciones y dotación de materiales, equipos, suministros, facilitación de los procesos al interior de la universidad, con la finalidad de contribuir al logro de su misión. La gestión administrativa es una expresión del logro de los objetivos de la institución, cuyo fin es facilitar los procesos de docencia, investigación y vinculación. Se establece la relación directa entre las funciones sustantivas de la universidad, con la gestión administrativa, debiendo caracterizarse por su eficiencia y eficacia. Es aquí en donde se puede evaluar cualitativamente el cumplimiento de planes, programas y proyectos, en función de un sistema de criterios, parámetros, variables e indicadores que permitan medir la calidad de los resultados.

Si se analiza a la organización como un sistema, se debe concluir que la gestión, debe entenderse como el proceso administrativo en sus 4 fases planificación, organización, dirección y control (Fayol 1916), buscando como integrarlas dentro de las funciones de la universidad, para en base a ello construir el plan estratégico, planes operativos, presupuestos y plan de acción.

Es necesario comprender que la gestión académica y administrativa universitaria se fundamenta en los postulados de las escuelas administrativas, y que, en relación al tipo de universidad imperante, le corresponde integrar o tomar elementos de una o varias de esas escuelas, para desarrollar una gestión que le permita acercarse al cumplimiento de sus objetivos y metas, procurando cumplir con su misión.

\section{Modelos de gestión universitaria}

Vargas (2013) indica que un modelo de gestión debe contener principios, enfoques, premisas, elementos previos, etapas fundamentales y 
fronteras. Al respecto, Sesento (2008) lo define como la representación de un hecho o fenómeno propuesto como ideal a seguir; que pretende mostrar la estructura de ese fenómeno, explicar sus elementos, mecanismos y procesos, su interrelación y los aspectos teóricos que le den sentido, para facilitar su comprensión. En tanto que Aguilera (2000), indica que el modelo es una representación parcial de la realidad; donde se puede dar explicación a un fenómeno o proceso específico, esto siempre desde el punto de vista de su autor. Vargas (2013) indica que la implementación del modelo exige una metodología o procedimiento que responda a las exigencias del mismo.

Los modelos de gestión universitaria, definidos dialécticamente en términos de administración para la eficiencia económica, administración para la eficacia pedagógica, administración para la efectividad política y administración para la relevancia cultural, son los elementos constitutivos de un paradigma de administración de la educación resultante de un esfuerzo superador de síntesis teórica de la experiencia latinoamericana de gestión educativa en el ámbito internacional. Solís et al., (2009) al referirse a los modelos universitarios indican que "no hay modelos puros, homogéneos, estables y carentes de contradicciones, ya que, por el contrario, suelen ser híbridos e inestables, cada universidad surge en respuesta a condiciones socio - históricas, geográficas, económicas y políticas particulares que determinan su modelo", criterio con el que comparto plenamente.

\section{Conclusión}

La construcción y reconstrucción del conocimiento en la educación y la gestión educativa comprometida con la calidad y la equidad implican un gran esfuerzo. Los países de América Latina, necesitan multiplicar, de modo urgente, sus conocimientos científicos y tecnológicos para que puedan participar activamente y beneficiarse equitativamente de la transformación política y económica en el mundo moderno, y buscar que no se amplié la brecha del desarrollo y la tecnológica, por lo que se plantea:

- La universidad latinoamericana debe adecuar sus estructuras y modelos de gestión a los desafíos de la sociedad posmoderna.

- Los sistemas de gestión de la calidad han sido ajenos a la realidad de las universidades, y los procesos de evaluación en Latinoamérica son recientes.

- La estructura por procesos permite adaptar las funciones universitarias a una moderna gestión administrativa.

Los modelos de gestión universitaria analizados y que se han dado a través de la historia presentan una serie de limitaciones entre las que están:

- No integran los procesos académicos y administrativos dentro de su estructura de funcionamiento.

- No se aprecia la forma en la que se toma en cuenta los elementos del 
entorno en el diseño de los modelos.

- No se hace visible un enfoque de procesos en el diseño de los modelos

- No se evidencia el papel de la planeación estratégica en el diseño de los modelos analizados.

- Los modelos estudiados son de carácter descriptivo, no se observa de forma gráfica su diseño.

- Los modelos evaluados no presentan un procedimiento adecuado para su implementación.

\section{References:}

1 Aguerrondo, I. (1993). La calidad de la educación: ejes para su definición y evaluación. Revista interamericana de desarrollo educativo, 37(116), 561-578.

2 Armengol Asparó, C., Castro Ceacero, D., Durán Bellonch, M. D. M., Essomba Gelabert, M. À., Feixas Condom, M., Gairín Sallán, J., ... \& Tomás Folch, M. (2009). La coordinación académica en la Universidad. Estrategias para una educación de calidad. Revista electrónica interuniversitaria de formación del profesorado, 29(12-2), 121-144.

3 Ayala Mora, E. (2014) "Entrevista a profundidad. Problemática de la Universidad Ecuatoriana y modelos de Gestión".

4 Banco Central Del Ecuador (1988). "Pensamiento Universitario Ecuatoriano”. Segunda Parte. Corporación Editora Nacional. Quito.

5 Braslavsky, C; Tiramonti, G. (1990). "Conducción educativa y calidad de la enseñanza media”. Editorial Miño y Dávila. 216 p. Buenos Aires.

6 Bruner, J. (2008). "El sistema de educación de Chile, un enfoque de economía política comparada". Revista da Avaliacao da Educao Superior. vol.13 número 2.

7 Cepal, N. (1972). Tendencias y estructuras de la economía de México en el último decenio.

8 Conferencia Mundial De Educación Para Todos (1990). "Jomtien, Tailandia", bajo el auspicio de UNESCO.

9 Cumbre de Jefes de Estado y de Gobierno de las Américas (1994). Miami

10 De Benito, C. (2000). La mejora continua en la gestión de calidad. Seis sigma, el camino para la excelencia. Economía Industrial, 331, p. 5966.

11 Fayol, H. (1916), “Administración industrial y general”. Francia

12 Freire, P. (2002). “Concientización: teoría y práctica de una educación liberadora, Buenos Aires”. Ediciones Galerna. 94 p. Primera edición.

13 Frigerio, G. Poggi M. Gianonni M. "Políticas, instituciones y actores en educación". (2000). 
14 Fuentes, H. (2003). La universidad y su gestión: una mirada dialéctico - holística”. Santiago de Cuba, CeeS Manuel F. Gran, Universidad de Oriente.

15 Galarza, J. (2007). "Modelo para evaluar la gestión de los procesos de planificación estratégica, información y evaluación de los objetivos en las Instituciones de Educación Superior adscritas al MES”, Tesis Doctoral, Universidad de la Habana, Cuba.

16 Gramsci, A. "Concepción dialéctica de la historia", Editorial Civilizacao Brasileira. (1987). 341 p. Rio de Janeiro.

17 Guerra Bretaña, R. Meizoso Valdés, M. (2012). Gestión de la calidad, conceptos, modelos y herramientas. Editorial UH. La Habana, Cuba.

18 Harry, M.; Schroeder, R. (2000). Six Sigma. Ed. Rosetta Books

19 Junta De Acreditación Nacional. (2009). Sistema de evaluación y acreditación de carreras universitarias, (SEA-CU). La Habana.

20 León R. (2008). Procesos de investigación y gestión, en la universidad de Oriente. Santiago de Cuba

21 León, R. (2012). Gestión universitaria y definición de políticas públicas. Universidad de Oriente.

22 Membrado, J. (2002). Curso Seis Sigma. Una estrategia de mejora. Qualitas Hodie, 95, p. 16- 21.

23 Namo De Mello, G. (2013). A pesquisa educacional do Brasil. Cuadernos de Pesquisa, publicacoes.fcc.org.br.

24 Noboa, P. (2012). Ensayo sobre educación superior". Jornadas de capacitación.

25 Sander, B. (2007). Revista de la Universidad de Chile, Perspectivas de la Educación Superior Chilena. pp. 45-62.

26 Schmelkes, S. (2006). La calidad de la educación y gestión escolar. Revista PRELAC, socolpe.org.

27 Sesento, L. (2008). Modelo sistémico basado en competencias para instituciones educativas públicas", Tesis Doctoral, Centro de Investigación y Desarrollo del Estado de Michoacán, México, disponible en www.eumed.net/tesis-doctorales/2012/lsg/index.htm.

28 Tadesco J.C. (1995). El nuevo pacto educativo: educación, competitividad y ciudadanía en la sociedad moderna. Editorial Anaya. Madrid.

29 UNESCO (a) (1996). "Conferencia Mundial sobre educación superior". Declaración mundial sobre la educación superior en el Siglo XXI: Visión y Acción. Paris.

30 UNESCO (b) (2013). "Clasificación Internacional Normalizada de la Educación Superior (CINE)". 